\title{
A design concept of fuzzy decision support system for construction workers safety monitoring
}

\author{
Lila Ayu Ratna Winanda ${ }^{1,3}$, Achmad Arifin ${ }^{2}$, Fauzan Arrofiqi ${ }^{2}$, Trijoko Wahyu Adi $^{1}$, and Nadjadji Anwar ${ }^{1}$ \\ ${ }^{1}$ Department of Civil Engineering, Institut Teknologi Sepuluh Nopember (ITS), Surabaya, Indonesia \\ ${ }^{2}$ Department of Biomedical Engineering, Institut Teknologi Sepuluh Nopember (ITS), Surabaya, Indonesia \\ ${ }^{3}$ Department of Civil Engineering, Institut Teknologi Nasional (ITN), Malang, Indonesia
}

\begin{abstract}
A construction project is very dynamic activities with high workloads and tight schedules, resulting in a fatigue condition of the workers. Due to the worker's physical and physiological conditions that change over time, the risks may be varying from fatigue, and may become a potential danger of accidents. In a modern construction work safety should be planned, monitored, analyzed easily using a sophisticated system. As an attempt to realize the system, our research group developed a decision support system for construction worker safety monitoring based on fuzzy system approach. The design concept of the fuzzy decision support system with focus on worker performance related to prediction of fatigue and safety is discussed in this paper. The decision support system was designed based on fuzzy inference system with physiological data (heart rate, body temperature, and muscle activity) and project environment (working space temperature) models. The knowledge about of human subject condition reflected by the physiological data, environmental conditions and their relationship with working performance and safety are formulated in a linguistic inference system. The realized system demonstrates the ability to evaluate worker performance during work hour and prediction of fatigue. The realized system is useful as an early warning system for the prevention of construction workers accident and a part of our attempt to realize the modern safety management system.
\end{abstract}

Keyword: Automation in civil construction, Construction worker, Fuzzy Decision Support System (FDSS), Safety Monitoring

\section{Introduction}

Health and safety in construction industry is still a problem marked by the highest number of construction accidents in almost all countries in the world including Indonesia compared with nother area [10, 32]. The uniqueness, dynamic and complex of construction project, so the accident can occur any time at working hours $[10,16]$.

Some regulation from the government has not been executed consistently by the project manager in project implementation [37]. While the manual check for H\&S leads the data not archived well and short time daily meeting, only discussed the possibility of hazards with no detail screenings for physiological condition.

Some previous studies show that the cause of constructions accident is dominant by human error which is unsafe act (80-90\%) and unsafe condition $(10 \%)$, We cannot be ignored the factors because there are relationships among these factors $[4,14,12,19,21$,
27]. Among the accidents due to human error, as much as $50 \%$ is caused by physical and psychological fatigue (internal factor of construction worker), so this condition must be a concern in the implementation of health and safety management system $[7,12,13]$. The environmental conditions of the project may also cause fatigue for workers, due to loss of alertness level and balance. Workers fatigue increases the risk of accident for construction workers because of performance degradation [10, 38].

Fatigue cannot be measured directly based on the source, can only be approached with the symptoms through physical and psychological condition of the construction workers [18, 22, 26, 35]. Generally, selfreport and subjective measurements are still used in most previous studies which discussed fatigue measurement for workers [8, 13, 22, 34, 35, 40]. Fatigue analysis with direct measurement, sensing and biomechanical measurement was applied in some studies, but they still used available devices separately. The lack of this

* Corresponding author: lila.winanda15@mhs.ce.its.ac.id 
approach results in a just in time measurement and cannot be combined with external factors outside of workers $[3,5,6,18,20,25]$. On the other hand, the dynamic construction work needs a fatigue analysis comprehensively based on internal and external factors.

The aim of this paper is to design a decision support system for real-time safety monitoring with a specific objective that focuses on construction workers fatigue. The design is a part of our goal in realizing modern safety management system that includes planning, monitoring and evaluation both in all subjects involved in construction project.

In our point of view, it is important to develop a safety monitoring system with a focus on workers fatigue as one of the causes accidents. Since fatigue is a physiological process, so it should be monitored in realtime. Therefore, we designed a conceptual fuzzy decision support system as a solution for real-time safety monitoring system for construction workers. A decision support system is much more sophisticated in decision system using human reasoning system, so we use fuzzy logic in this approach because assessment, evaluation and decision in construction safety for construction workers require the assessment of qualitative human expertise and are difficult to put in an exact value. Fuzzy logic approach has been applied in many areas thus it is minimizing the disadvantages of the qualitative approach when there is subjectivity, doubt and expert judgment $[11,17,18,23,39]$.

This report is organized as follows section 2 describes the methodology of the paper which is divided into concept of design, fuzzy decision support system and computer simulation studies. Section 3 presents the result and explains the findings. Section 4 includes a discussion around the result, limitation and future research. Section 5 is conclusion of the paper.

\section{Method}

Physical fatigue prediction for worker can be done based on the factors that identify the symptoms of a worker's fatigue. In terms of real-time monitoring system, realtime measurable indicators can be used as approaches, such as heart rate, body temperature, muscle activity and working space temperature. These indicators can directly show the symptoms of fatigue.

Moreover, measurable change in real-time has indicator that is also influenced by other discrete factors, such as workers' characteristics (age, height, weight and lifestyle) and workload to be completed. These factors indirectly contribute to identify a worker's physical fatigue. The correlations among the existing factors are arranged in causal diagram as defined in the previous study $[1,36]$.

\subsection{Concept of Design}

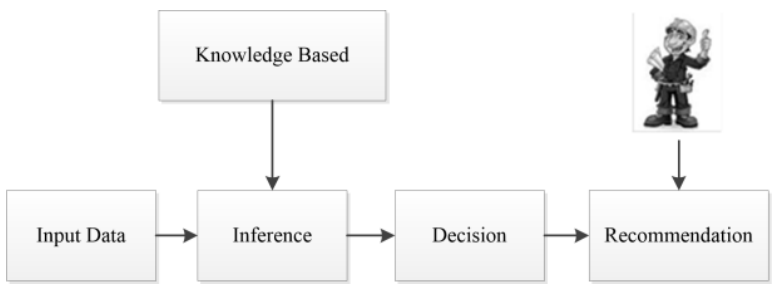

\subsubsection{Decision Support System (DSS)}

Developing a decision support system needs an input data for inference state. According to knowledge based from expertise, experience or database, the inference processed to achieve a decision as recommendation basis for user to be implemented. A complete scheme is shown in Figure 1.

\subsubsection{Development Safety Monitoring System}

The development of monitoring system for workers needs some phases, starting from variables identification of construction safety and fuzzy computation scheme is used for analysis (Figure 2). To support the analysis process, it requires a software development. For this research, we use Delphi 7.0 with the basic programming language is Pascal. The experimental test can be done from the developed system, is useful for assessment and suggestion phases as feedback for next improvement.

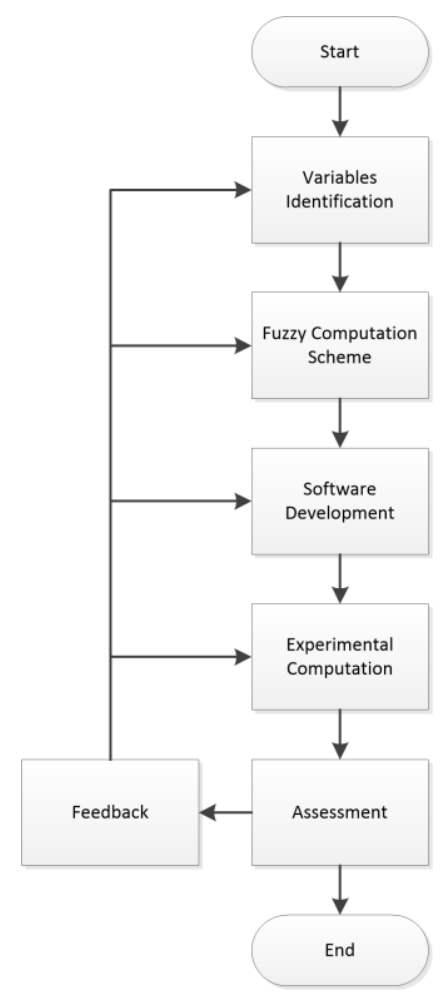

Fig. 2. Development of Decision Support System

\subsection{Variables Identification}

There are several variables inputted in this developed system, such as heart rate, body temperature, muscle activity and working space temperature while the output variables the output variable is one of the variable, i.e., performance or fatigue prediction in which indicates the physical condition of construction workers. 


\subsection{Heart Rate}

Heart rate is the most commonly used indicator and answer many of the problems of human physiology in static and dynamic states during working time [15, 24]. Based on the American Heart Association, mentioned that the normal heart rate ranges from 50 to $100 \mathrm{bpm}$ but the working heart rate ranges between $90-130 \mathrm{bpm}$ is recommended to support performance [2]. When someone gets on work, the heart rate can be changes. It caused by several factors such as age, weight, body temperature, work load and lifestyle [33].

\subsubsection{Body Temperature}

Escalation in body temperature that can be lead to heat exhaustion is a signal that the body becomes overheated. Normal body temperature is $36.5^{\circ} \mathrm{C}-37.2^{\circ} \mathrm{C}$ [43] Hypothermia is when the body temperature is $<35^{\circ} \mathrm{C}$ and hyperthermia occurs when the body temperature is $>$ $40^{\circ} \mathrm{C}$.

\subsubsection{Muscle Activity}

Muscle activity can be assessed based on the surface electromyogram (sEMG) and the maximum voluntary contraction (MVC) [29, 44], sEMG normalized to MVC (\%MVC) is an indicator that can describe muscle tension and muscle fatigue. The EMG signal character has relatively small amplitude around $10 \mathrm{mV}$ with a frequency range $20 \mathrm{~Hz}-500 \mathrm{~Hz}$ [9]. A bioinstrumentation with high gain and band pass filter with $\mathrm{BW} 20 \mathrm{~Hz}_{-}^{-}$ $500 \mathrm{~Hz}$ is usually realized to measure the signal. A particular processing method (analog or digital environment) is also performed to obtain linear envelope of the measured signal, followed by normalization with MVC [28]. The other method of fatigue analysis using raw sEMG signal transformed to frequency domain. The decrement of frequency is an indicator of fatigue [28].

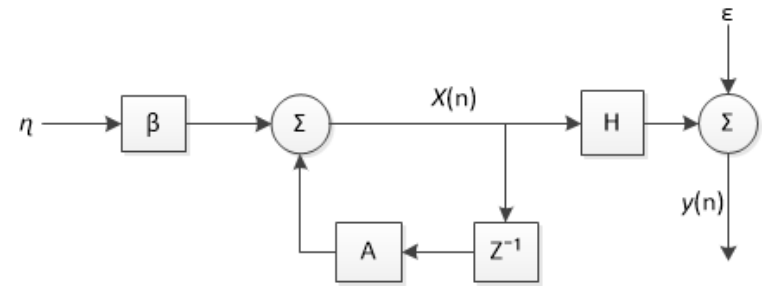

Fig. 3. State space model of time variant input variables

\subsubsection{Working Space Temperature}

The regulation from Indonesian government mentions that the comforTable temperature in Indonesia is $26.7^{\circ} \mathrm{C}$. According to Suma'mur in the quotation of [30], for Indonesian worker, the comforTable weather is in $21^{\circ} \mathrm{C}$ $30^{\circ} \mathrm{C}$. The rising of environment temperature will increase the body temperature and stimulate out sweat which contains sodium chloride. Reduced levels of sodium chloride in the body will inhibit transportation of glucose as an energy source resulting in decrease levels of muscle contraction and cause fatigue [30].

\subsubsection{Workers Performance and Fatigue Prediction}

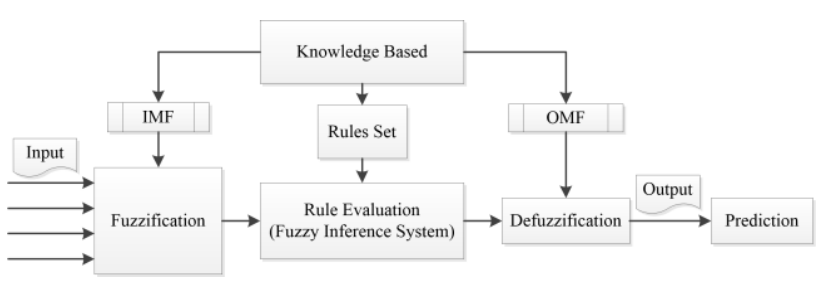

Fig. 4. The global concept of fuzzy system

In safety prevention program, workers monitoring system is applied before and during working time, in our research it is called performance and fatigue prediction. The percentage of performance (\%) is the readiness measure of workers physical condition before started the task. The higher, the better preparation to work and otherwise is indicated the more vulnerable physical condition to fatigue.

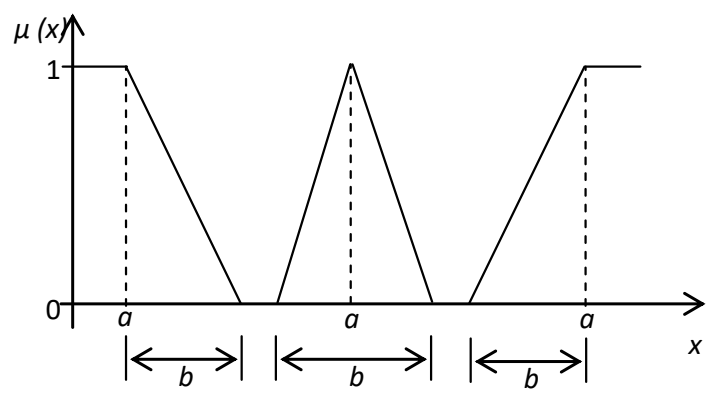

Fig. 5. Defining membership function

According to Tarwaka in [7] mentioned that fatigue can reduce performance, increase human errors and workplace accident. There is no agreement on the scale of fatigue assessment and each study uses a different scale related to the contradiction in the subjectivity of judgement between one person and another [2]. Using a subjective measurement, fatigue condition usually occurs at the end of working times if the average workload exceeds $30-40 \%$ of the maximum aerobic power [34, 36].

\subsection{Realization of a Fuzzy DSS For Safety Monitoring}

All input variables that are changed over time are modeled using state space signal generation as shown in Figure 3. The state space model represents the dynamic of signal process and an observation equation models noisy observation signal $[41,42]$.

Fuzzy decision support system is structured to monitor the safety of constructions workers in real-time during working hours, especially for fatigue. Generally, the fuzzy logic process begins with input/output variables identification and changes into linguistic variables. Then, the inference engine on fuzzy system will define the expected output based on the rules aggregation and changes into crisp value as final output 
of all the fuzzy process. The global concept of fuzzy system is shown in Figure 4.

The first step in fuzzy analysis is fuzzification ( change the crisp value into the fuzzy input/linguistic variable), which is determined by its membership function through a function approach (triangular function, trapeziodal function, etc.). The input and output variables are divided into several membership functions. Triangular fuzzy number (TFN) is used as a membership function in this research, due to widely applied in previous studies and shows a high precision result. (Figure 5).

Mathematical formulations for down linear function:

$\mu(x)= \begin{cases}1, & x \leq a \\ (a+b)-x /(a+b)-a, & a<x<(a+b) \\ 0, & x \geq(a+b)\end{cases}$

Mathematical formulations for triangular function:

$\mu(x)= \begin{cases}0, & x \leq(a-1 / 2 b) \\ x-(a-1 / 2 b) / a-(a-1 / 2 b), & (a-1 / 2 b)<x<a \\ (a+1 / 2 b)-x /(a+1 / 2 b)-a, & a \leq x<(a+1 / 2 b) \\ 1, & x=a\end{cases}$

Mathematical formulations for up linear function:

$\mu(x)= \begin{cases}0, & x \leq(a-b) \\ x-(a-b) / a-(a-b), & (a-b)<x<a \\ 1, & x \geq a\end{cases}$

In fuzzy system, the inference process considers all the rules in accordance with knowledge-based. In syntax, a fuzzy rule is written as follows:

\section{IF antecendent THEN consequent}

Fuzzy inference system works by Mamdani method (min - max method). Using this method, to achieve the output, it requires four stages: creating fuzzy sets, applied the implication functions (rules aggregation) using min function and rule composition using max function in inference engine. A fuzzy rule is defined as follow:

IF $\mathrm{x}_{1}$ is $\mathrm{A}_{1}$ AND ... AND $\mathrm{x}_{\mathrm{n}}$ is $\mathrm{A}_{\mathrm{n}}$ THEN $\mathrm{y}$ is $\mathrm{B}$

with $A_{1}, \ldots, A_{n}$, and $B$ are linguistic variables (fuzzy sets) and " $x_{1}$ is $A_{1}$ " stated that the value of $x_{1}$ variable is the member of fuzzy set $A_{1}$ (Figure 6).

In Mamdani method, implication function using AND operator obtained by using MIN function. The function of MIN is the function to find the smallest membership value from two or more operand. Generally, it can be drawn as following equation [31]:

$$
\mu A \cap B=\min (\mu A[x], \mu B[y])
$$

Analysis of rule composition uses MAX function by taking maximum value of rule, then using it to modify fuzzy area and applying it to output. Generally, the calculation of rule composition can be drawn with the equation of:

$$
\mu \mathrm{sf}(\mathrm{xi})=\max (\mu s f(x i), \mu k f(x i))
$$

where $\mu s f\left(\mathrm{x}_{\mathrm{i}}\right)$ is the value of fuzzy membership solution until $i$ rule, and $\mu k f\left(\mathrm{x}_{\mathrm{i}}\right)$ is the value of $i$ rule of consistent fuzzy membership.
Futhermore, based on the fuzzy rules that have been prepared, the next step is rule evaluation which is the process of rule's selection and represents the degree of membership value from a defined linguistic variable. (Figure 7).

The last step in fuzzy analysis is defuzzification. The input of defuzzification process is a fuzzy set obtained from the fuzzy rules composition. The final output is a number in the fuzzy set domain, so if a fuzzy set is given within a certain range, the crisp value will obtained as an output.

In this research, defuzzification used centroid method as commonlly used in fuzzy Mamdani (Figure 8). Centroid method takes central point $\left(Z^{*}\right)$ of fuzzy area as crisp solution through following equation:

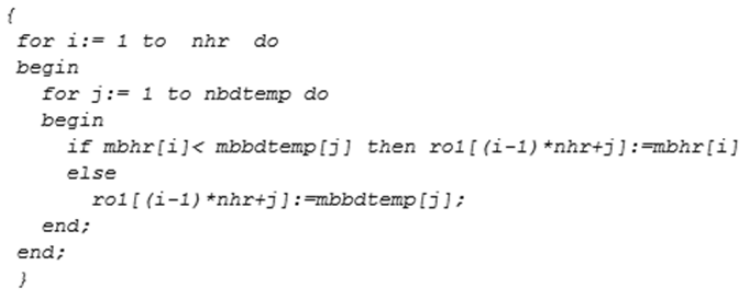

Fig. 6. Rule Identification in Input and Output Variables

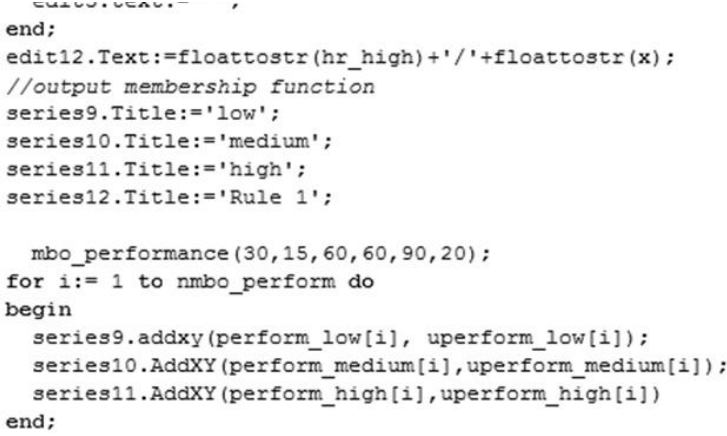

Fig. 7. Rules Evaluation

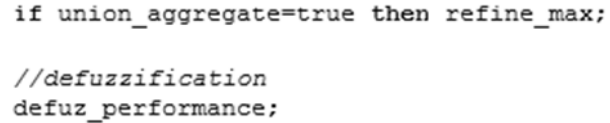

Fig. 8. Defuzzification Formulation

$$
z^{*}=\frac{\sum \mu(z) \cdot z}{\sum \mu(z)}
$$

\subsection{Computer Simulation Studies}

Software development aims to build a decision support system application program for safety monitoring of construction workers. The application program contains several menus related to the workers performance and safety monitoring (Figure 9). 


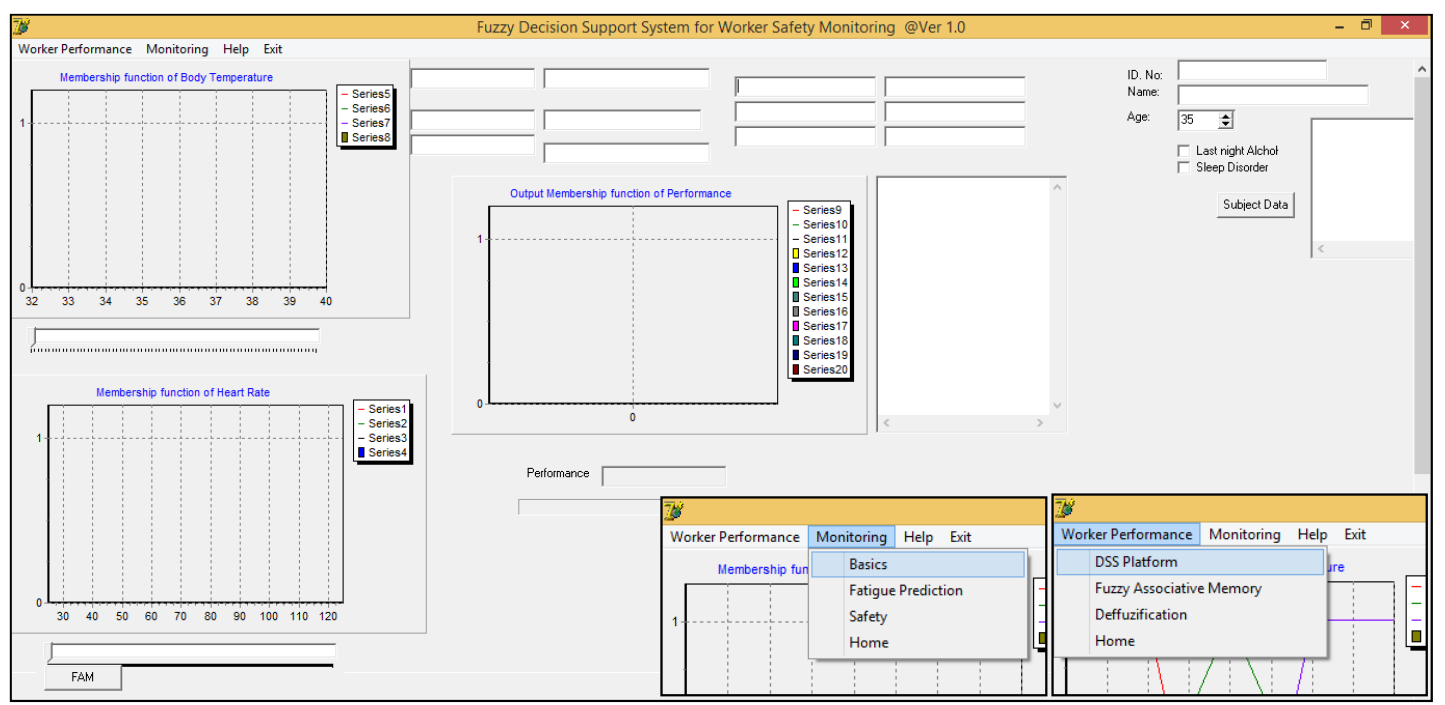

Fig. 9. Main Menu of Fuzzy Decision Support System

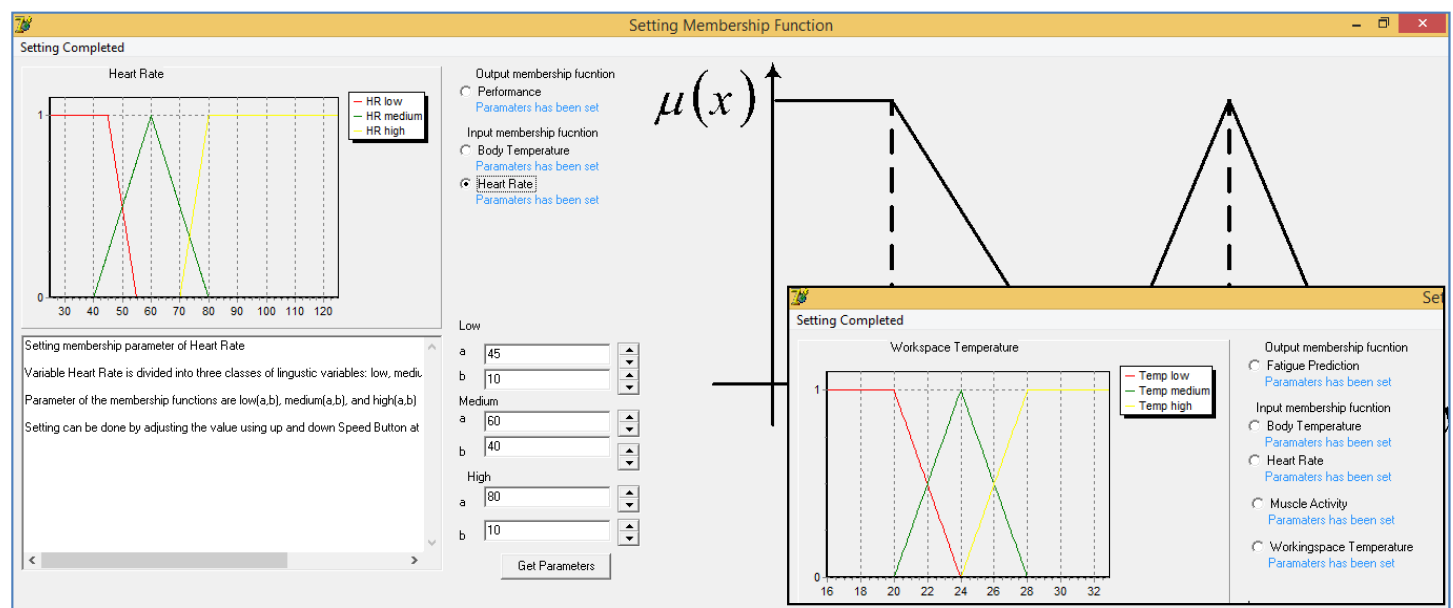

Fig. 10. Setting Menu of Membership Function

The main menu is developed to accommodate the analysis of data acquisition from the input and output variables, which is represented in each membership function. The sub-menu is supporting the steps in fuzzy analysis In this research, the discussion is limited to the fatigue prediction monitoring system (Figure 10).

The DSS platform is useful as an initial screening before starting work, is only using internal factors. Meanwhile, the menu 'workers performance' to show how the developed fuzzy system is running well. Fuzzy association memory menu shows the rules set based on the input data and defuzzificaton menu as the last process in fuzzy system (Figure 11).

Fatigue prediction monitoring is structured to represent how the monitoring system of construction worker fatigue during work hours. The monitoring system is developed in real-time to show the alteration of workers condition. The 'running' system in fatigue prediction is shown in real-time during working times of construction workers.

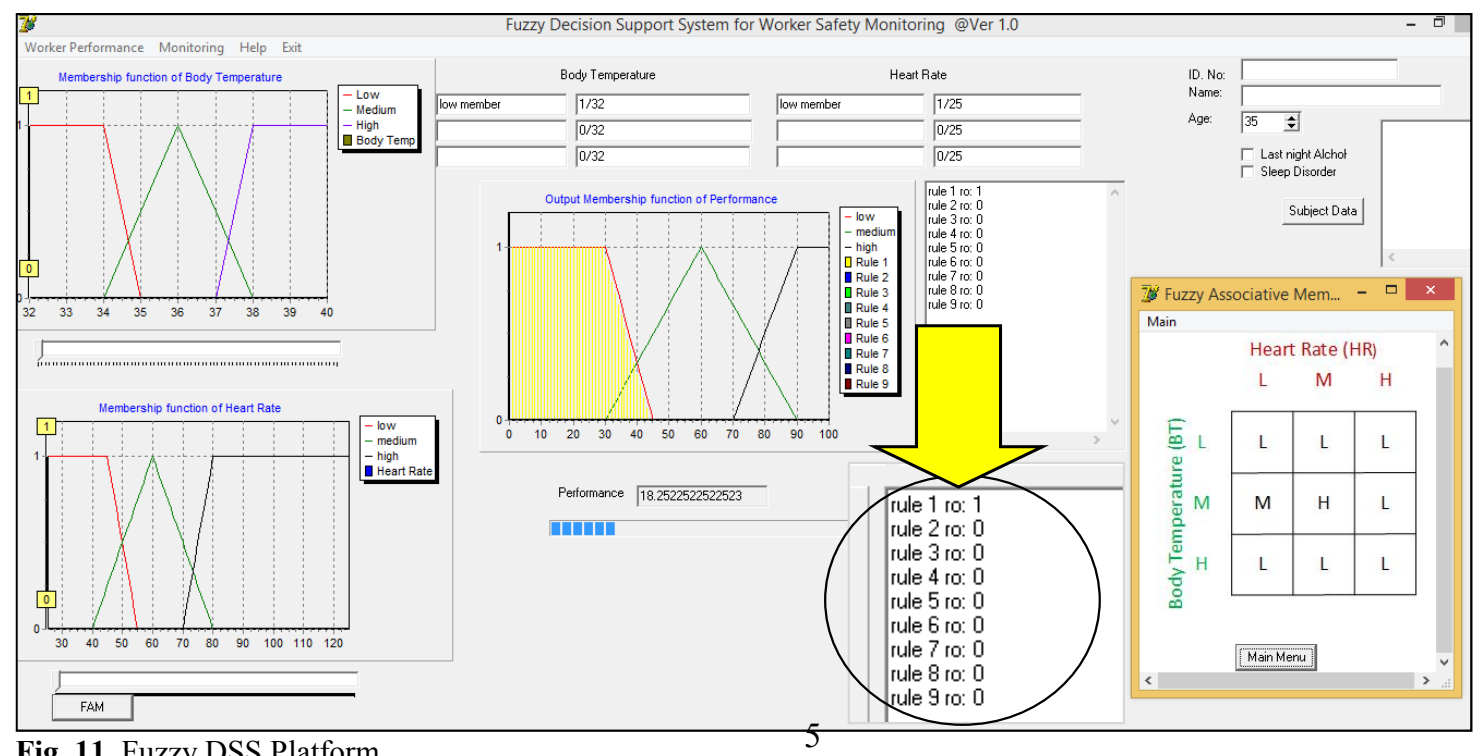




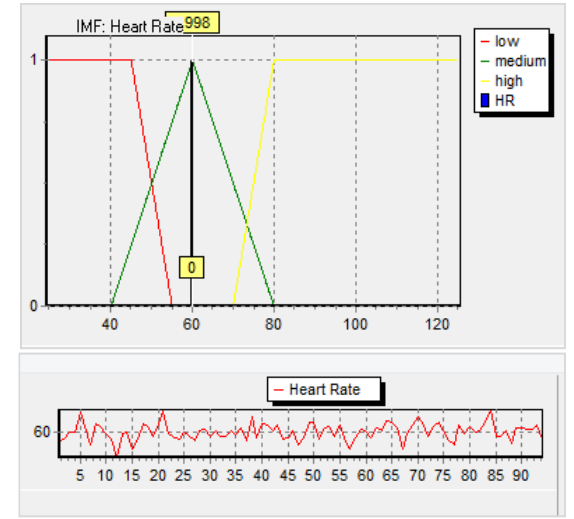

Fig. 12. Heart Rate Value

\section{Results}

The fuzzy decision support system (FDSS) in this report is developed into two parts: DSS platform and fatigue prediction analysis as a part of a modern safety monitoring system in real-time.

\subsection{Fuzzy DSS Platform}

The DSS platform shows the physiological conditions of construction workers when they get prepared to start the task. The indicators are heart rate and body temperature with the output variable is performance.

Using membership function setting menu, the input variables will be selected and edited according to the expertise, experience and database identification. In accordance with the input variables identification, the rules are arranged for fuzzy inference process as shown in fuzzy association memory with nine rules for fuzzy DSS platform.

The system will automatically display the class category of membership function, when we drag the option value of heart rate and body temperature. Using the rules set, the system automatically runs the inference process. Then, the system will select and displays the winning rule as zoomed in Figure 11. The result of rule evaluation on monitoring system gives the output category of performance and through the defuzzification process, will automatically converts the value into crisp as a final output. In DSS platform, the indicators are used to analyze the performance of construction workers. The higher number of performance means the readiness of physical condition to work.

\subsection{Fatigue Prediction}

When the worker condition is ready to work based on the value of its performance, real-time monitoring during working hours can be done to analyze the fatigue condition. Doing job activity for worker means that there are some additional factors to describe the fatigue condition such as external variables that is also play a role, in addition to internal variables.

To accommodate the needs in fatigue prediction analysis, the input variables are divided into internal variables (heart rate, body temperature and muscle activity) and external variables (working space temperature). The input data is shown in Figure 15.

The real-time input variables describe the acquisition data from the sensor network which changes over time. However, for this study, real time input was generated from an auto regressive data to represent the sensor system as a part of our future work (Figure 12).

According to knowledge-based, fuzzy inference system is processed on the fatigue analysis through the identification of 81 rules set for rule evaluation (Figure 13). The output variable is a real-time fatigue prediction monitoring for construction workers (Figure 14). The result of monitoring system for fatigue prediction is shown in Figure 15.

$$
\begin{array}{|l}
\text { rule } 41 \text { ro: } 0.456357540570652 \\
\text { rule } 42 \text { ro: } 0.000253993569259592 \\
\text { rule } 44 \text { ro: } 0.326185475657609 \\
\text { rule } 45 \text { ro: } 0.000253993569259592
\end{array}
$$

Fig. 13. The selected rules in fatigue prediction

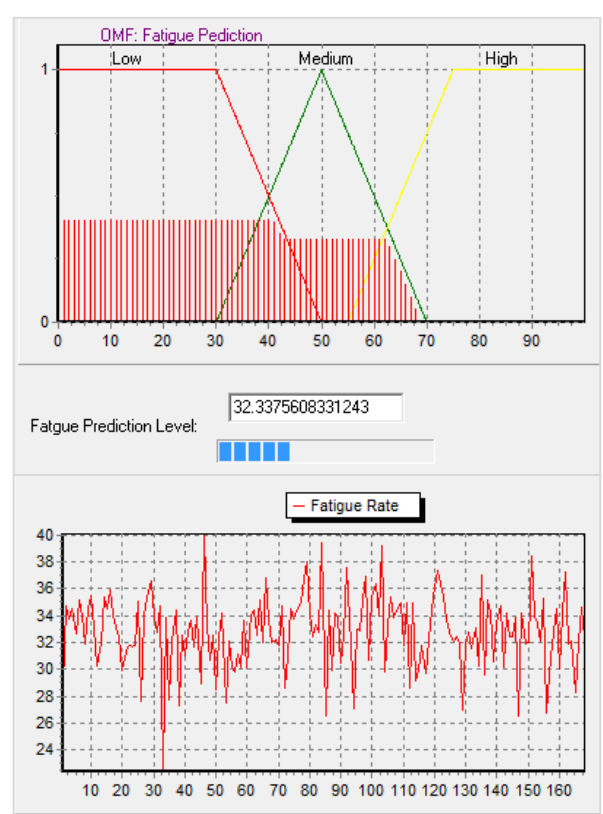

Fig. 14. The output of fatigue prediction

The result of real-time monitoring system in this study is fatigue prediction which shows the fatigue condition of construction workers during working hours.

The higher rate of fatigue prediction means the worse condition of construction workers to continue their activities. It means the hazard level of workers is in higher possibility of working accidents. 


\subsection{Computational Experiment Results}

category through the defuzzification process. The result

Computational experiment is attempted on the DSS of the developed system is the same as the manual

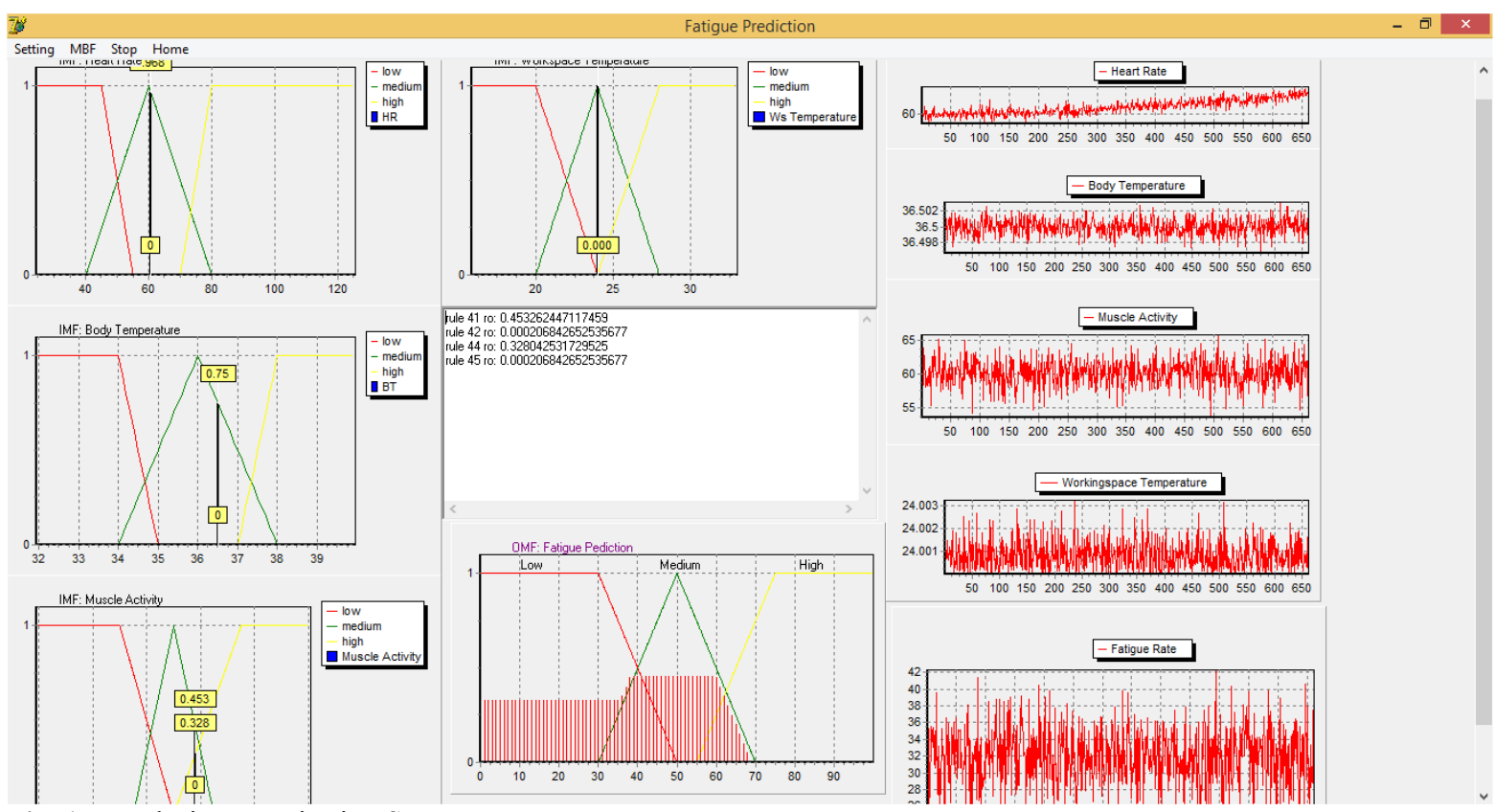

Fig. 15. Real Time Monitoring System

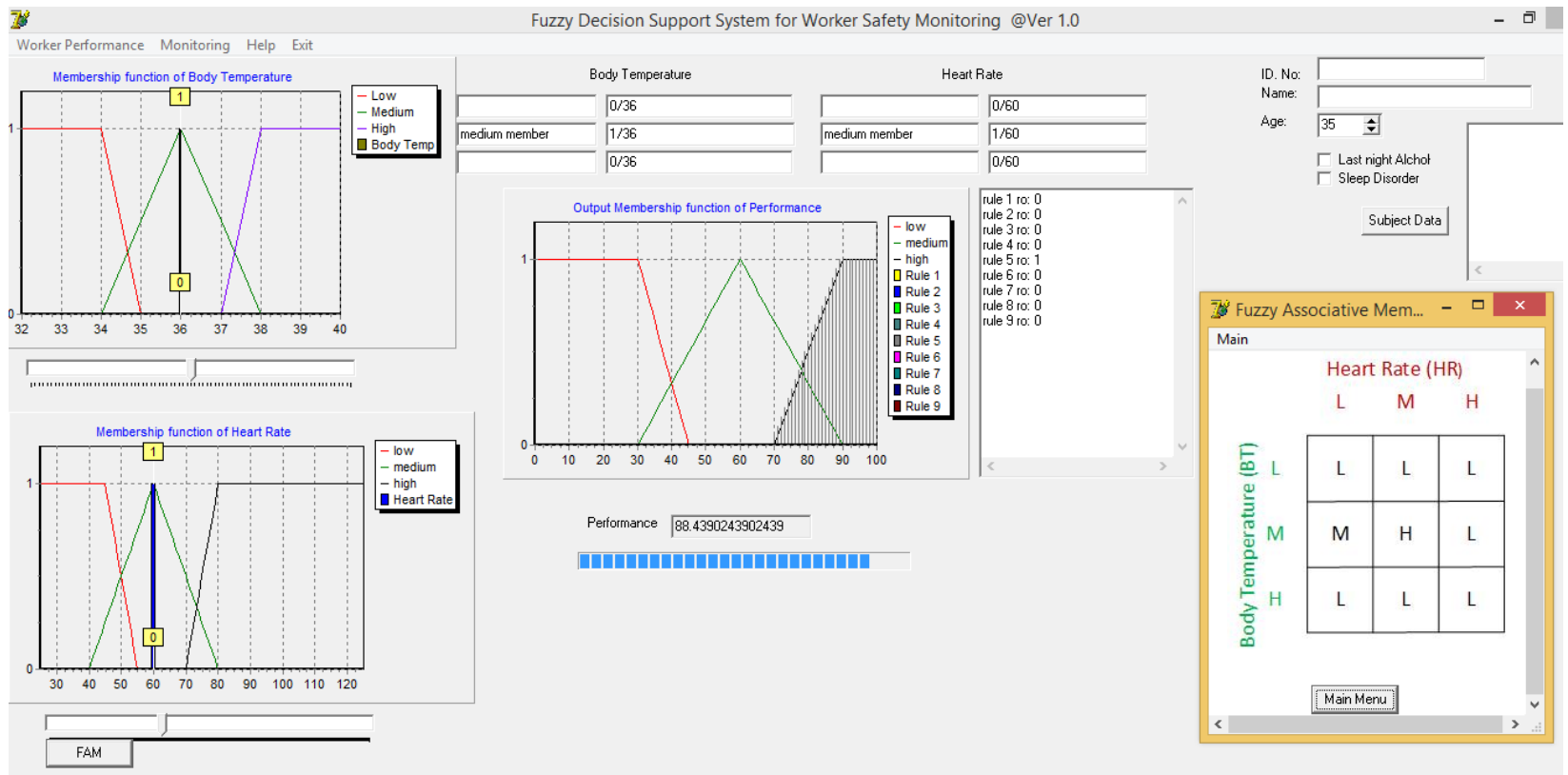

Fig. 16. DSS platform checking system

platform to test and evaluate the developed fuzzy system. We tested the DSS platform system using the data of heart rate $=60 \mathrm{bpm}$ and body temperature $=36^{\circ} \mathrm{C}$. The system reads automatically that the heart rates category and the body temperature is medium. The system shows the performance category is high with $88.439 \%$.

We evaluate the result through the rules set manually, IF $\mathrm{HR}$ is medium AND BT is medium THEN the performance is high. As shown in fuzzy association memory (Figure 16), the system identified the winning rule is number 5, IF HR is medium AND BT is medium THEN performance is high. Meanwhile, the result of performance with $88.439 \%$ is suiTable for the high analysis. The test of overall DSS platform system showed that the classification of performance through fuzzy reasoning has already worked well and proper.

The reliability of fuzzy system is checked through data simulation from construction worker's heart rate is $73.9 \mathrm{bpm}$ and body temperature is $37.3^{\circ} \mathrm{C}$. According to the data, the class classifications are medium and high to its membership function. When the rule evaluation is operated using min-max function of Mamdani method, it was found 4 rules from fuzzy association memory, i.e.:

a. Number 5 (If HR is $\mathrm{M}$ and $\mathrm{BT}$ is $\mathrm{M}$ then $\mathrm{P}$ is $\mathrm{H}$ ) with the degree of membership $=0.305$

b. Number 6 (If HR is $\mathrm{M}$ and $\mathrm{BT}$ is $\mathrm{H}$ then $\mathrm{P}$ is $\mathrm{L}$ ) with the degree of membership $=0.35$ 


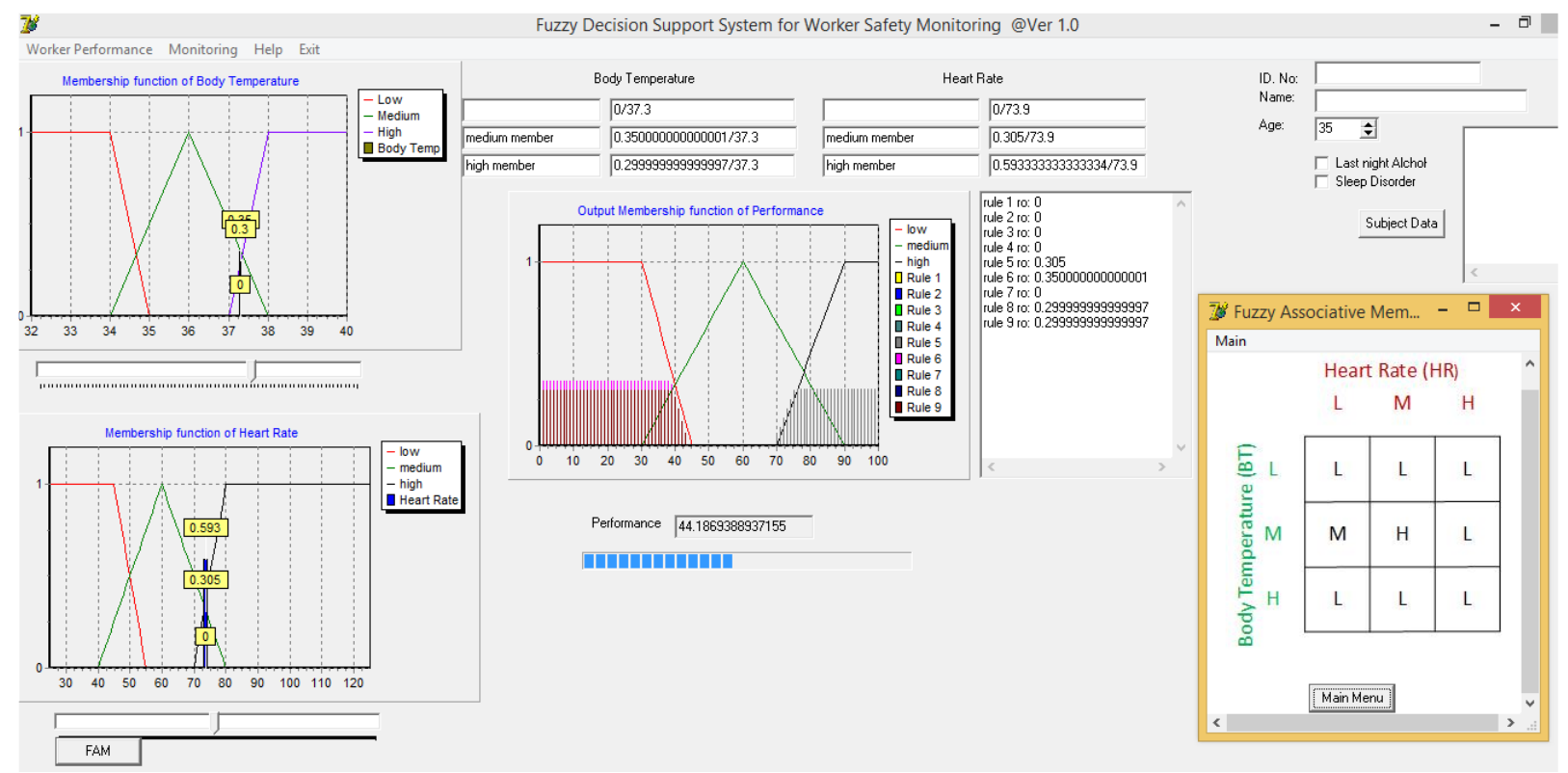

Fig. 17. Simulation on DSS Platform

c. Number 8 (If $\mathrm{HR}$ is $\mathrm{H}$ and $\mathrm{BT}$ is $\mathrm{M}$ then $\mathrm{P}$ is $\mathrm{L}$ ) with the degree of membership $=0.299$

d. Number 9 (If $\mathrm{HR}$ is $\mathrm{H}$ and $\mathrm{BT}$ is $\mathrm{H}$ then $\mathrm{P}$ is $\mathrm{L}$ ) with the degree of membership $=0.299$.

The output variable, performance is in medium and low category. Through the defuzzification mechanism, then we obtained the crisp value of performance is $44.18 \%$. The completed process is shown in Figure 17.

For the real-time monitoring system, it can be seen in the application window that the input and output data are proceed based on the time function. A workers activity, means taking the workload to be assigned and it indicate the changing in the physiological condition. In accordance with some previous studies mentioned that the increased workload can raise the heart rate during working hours (Figure 18).

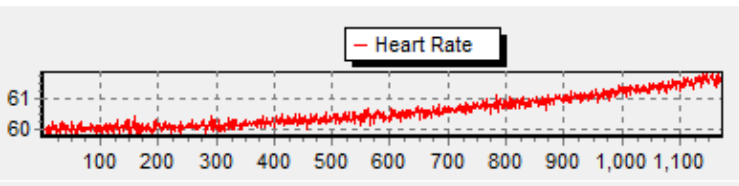

Fig. 18. The Changes of Heart Rate During Work

An autoregressive generated data was used to check the reliability of the system through experimental simulations. The simulation for fatigue prediction was tested under a normal range condition of worker (body temperature $(\mathrm{BT})=36.5^{\circ} \mathrm{C}$, working space temperature $(\mathrm{WST})=24^{\circ} \mathrm{C}$ and muscle activity (MA) response $=$ $60 \%$ ) with the heart rate (HR) value started at normal

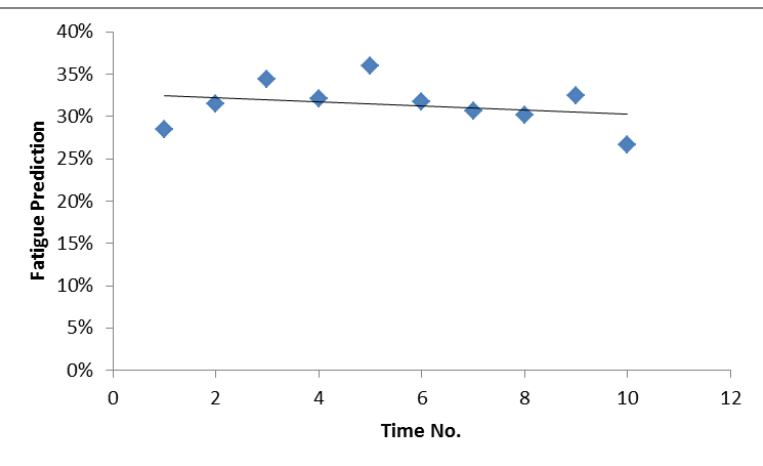

Fig. 19. Fatigue Prediction in Normal Condition

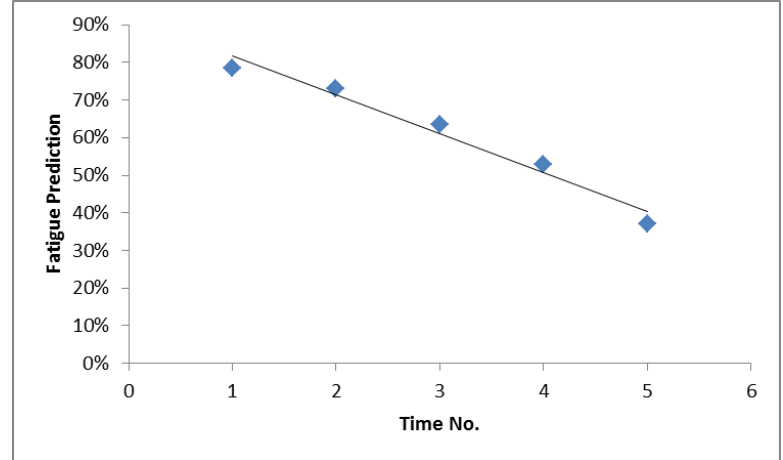

Fig. 20. Fatigue Prediction in Up-normal Condition

condition $=60 \mathrm{bpm}$. The process of fuzzy analysis was the same with the DSS simulation. The results are presented in Table 1 and Figure 19.

Table 1. Real-time Monitoring Simulation in Normal Condition

\begin{tabular}{|c|c|c|}
\hline Time No. & Winning Rules & Fatigue Prediction (F) \\
\hline 1 & $41,42,44,45$ & $28.458 \%$ \\
\hline 2 & $41,42,44,45$ & $31.522 \%$ \\
\hline 3 & $41,42,44,45$ & $34.337 \%$ \\
\hline 4 & $41,42,44,45$ & $32.095 \%$ \\
\hline 5 & $41,42,44,45$ & $35.974 \%$ \\
\hline
\end{tabular}


Based on the Table 1, it was found that rule number $41,42,44$ and 45 have been selected from 81 rules in fatigue analysis, i.e.:

a. Number 41 (If HR is $\mathrm{M}$ and $\mathrm{BT}$ is $\mathrm{M}$ and MA is $\mathrm{M}$ and WST is $M$ then $F$ is $M$ )

b. Number 42 (If HR is $\mathrm{M}$ and BT is $\mathrm{M}$ and MA is M and WST is $\mathrm{H}$ then $\mathrm{F}$ is $\mathrm{M}$ )

c. Number 44 (If HR is $\mathrm{M}$ and $\mathrm{BT}$ is $\mathrm{M}$ and $\mathrm{MA}$ is $\mathrm{H}$ and WST is $\mathrm{M}$ then $\mathrm{F}$ is $\mathrm{L}$ )

d. Number 45 (If $\mathrm{HR}$ is $\mathrm{M}$ and $\mathrm{BT}$ is $\mathrm{M}$ and $\mathrm{MA}$ is $\mathrm{H}$ and WST is $\mathrm{H}$ then $\mathrm{F}$ is $\mathrm{M}$ )

The output shows that the fatigue prediction was in lowmedium category, while according to the Figure, it can be shown that the average value of fatigue prediction during normal range condition is $31.399 \%$. It means, if the worker is in normal condition, the output system will show the low-medium category for the fatigue prediction and the workers is in safe condition to continue their job.

In other case where it started with a low category of heart rate, it can be shown that the winning rules were changing over time. The result is shown in Table 2 and Figure 20.

Table 2. Real-time Monitoring Simulation in Up-normal Condition

\begin{tabular}{|c|c|c|}
\hline Time No. & Winning Rules & Fatigue Prediction (F) \\
\hline 1 & 14,17 & $78.585 \%$ \\
\hline 2 & $\begin{array}{c}5,8,14,17,32, \\
35,41,44\end{array}$ & $73.022 \%$ \\
\hline 3 & $14,17,41,44$ & $63.489 \%$ \\
\hline 4 & $14,17,41,44$ & $52.883 \%$ \\
\hline 5 & $32,35,41,44$ & $37.022 \%$ \\
\hline
\end{tabular}

From the Table 2 and Figure 20, it looks that the fatigue prediction is decreasing from high to low condition because the low number of heart rate causes the worker to feel very tired. The fatigue condition will decline together with the increasing of heart rate at normal value (based on Figure 18) and it will reduce the fatigue.

\section{Discussions}

An expected system to support a decision making of construction worker safety has been realized. The fuzzy logic approach is used to analyze the safety variables related to performance and fatigue prediction. Through the analysis steps (variables identification, development of fuzzy decision support, simulation and experimental computation), the resulting system is appropriate.

Based on the testing results of the DSS platform, it can be concluded that the model is able to demonstrate the performance of fuzzy system based on the knowledge based. The model can be used to find out the readiness of workers in preparation stage using the physiological indicators of construction workers through the performance variable.

According to the fatigue prediction analysis, the system has been able to present input data in real-time and predicted workers fatigue during the specified working period as an output system.

The realized system should be developed more as future research, such as applying another type and numbers of membership function to find out the effect of variables and simulate some treatments to the system (rest time, overtime and additional workload). As a part of final goal of our research, it still needed to combine the software and hardware system as an integrated early warning system for construction safety.

\section{Conclusions}

We have established a fuzzy decision support system for real-time monitoring of construction workers safety. System design was realized base on approach of fuzzy system realization.

From the developed system which is divided into the DSS platform and fatigue prediction simulation, fuzzy logic denotes as a satisfactory system in real-time safety monitoring for construction workers. It is shown through the mechanism process running well from input data, inference system until the final output.

The simulation experimental which is shown by the developed system, can describe the workers condition and its changes during the work in accordance with the knowledge-based. Fatigue analysis in this research was done using objective measurement (from in-depth measurement of supporting indicators) so it has a higher accuracy than subjective measurement (from self-report expression)

The final output of FDSS is useful as recommendations in decision making related to worker safety conditions before and during work through fatigue prediction. The current realized system would be integrated with hardware system to realize the final goal, a modern safety monitoring as an application of automation in construction project platform.

This research was supported by Beasiswa Pendidikan Pascasarjana Dalam Negeri (BPPDN) grant fiscal year 2015. The authors also wish to acknowledge the contributions of medical electronic laboratory, which has given support for this research.

\section{References}

1. T. J. Adi, L. A. Winanda, EACEF 2017, 1-7 ( MATEC Web of Conferences 138, 2017)

2. A. Aryal, A. Ghahramani, B.B. Gerber, Autom. Constr. 82, 154-165 (2017)

3. F. L. Chang, Y. M. Sun, K. H. Chuang, D. J. Hsu, Appl. Ergon, 1-6 (2008)

4. A. Charehzehi, A. Ahankoob, IJAET, 303-312 (2012) 
5. T. Cheng, G. C Migliaccio, J. Teizer, U. C. Gatti, J. Comput. Civ Eng. 27, 320-335 (2013)

6. H. Dong, I. Ugalde, N. Figueroa, A. E. Saddik, Sensors 14, 2052-2070 (2014)

7. M. Fadel, M. Muis, S. S. Russeng, (http://repository.unhas.ac.id) (2016) (2014)

8. D. Fang, Z. Jiang, Z. Mingzong, W. Han, Safety Science 73, 80-91(2015)

9. S. Firdaus, M. Adriana, Jurnal Elemen 3 (1), 18-22 (2016)

10. Y. M. Goh, N. F. Sa'adon, J. Constr. Eng. Manage, 141 (16), (2015)

11. M. Gunduz, T. Birgonul, M. Ozdemis, J. Constr. Eng. Manage (2017)

12. S. Han, S. Lee, F. P. Mora, J. Comput. Civ. Eng 27 (6), 635-644 (2013)

13. M. Haumaru, Aotearoa, Safety, Health and Welfare on Construction Site (2014)

14. S. S. Hosseinian, Z. J. Torghabeh, IJAET, 53-66 (2012)

15. K. Hu, H. Rahmandad, T. Smith-Jackson, W. W. Winchester, Construction Management and Economic 29, 397-416 (2011)

16. S. Isaac, T. Edrei, Autom. Constr 63, 66-78 (2016)

17. M. Javadi, G. Saeedi, K. Shahriar, J. Appl. Sci 17, 103-115 (2017)

18. N. Javaid, S.Faisal, Z. Khan, D. Nayab, M. Zahid, IEEE 8th International Conference on Broadband and Wireless Computing (Cornell University Library, Compeigne, France, 2013)

19. Z. Jiang, D. Fang, M. Zhang, J. Manage. Eng. 31 (6) (2015)

20. J. T. Kider, K. Pollock, A. Safonova, Eurographics/ ACM SIGGRAPH Symposium on Computer Animation (Association for Computing Machinery Inc., 2011)

21. H. Li, G. Chan, T. Huang, M. Skitmore, T. Y. E. Tao, E. Luo, J. Chung, X. S. Chan, Y. F. Li, Autom. Constr. 55, 58-65 (2015)

22. N. Y. Limbong, J. Josephus, P. A. Kawatu, (http:// fkm.unsrat.ac.id) (2016) (2015)

23. H. T. Liu, Y. I. Tsai, Safety Science, 1067-1078 (2012)

24. I. K. Manurung, J. TIN Universitas Tanjungpura 1(2) (2013)

25. F. Nde, J. Nebo, W. Ngatchou, C. Tchatchoua, A. M. Sone, C. D. Brouwer, Pan African Medical Journal (2013)

26. C. O'Neill, K. Panuwatwanich, Proceedings of the 4th International Conference on Engineering Project, and Production Management (EPPM 2013)( pp. 993-1005, 2013)

27. D. A. Patel, K. N. Jha, J. Constr. Eng. Manage. 14 (1) (2015)

28. J. Perry, J. M. Burnfield,. Gait Analysis : Normal and Pathological Function, Second Edition. (SLACK Incorporated, 2010)

29. J. S. Petrofsky, R. M. Glaser, C. A. Phillips, Ergonomics 25 (3), 213-223 (1982)

30. I. M. Ramdan, The Indonesian Journal of Public Health 1 (2007)
31. Rodiah, R. Widodo, Seminar Nasional Teknologi Informasi dan Komunikasi Terapan (SEMANTIK) (p. 471, 2015)

32. H. Russell, B. Maitre, W. Dorothy, Trends and Patterns in Occupational Health and Safety in Ireland (The Economic and Social Research Institute, Dublin , 2015)

33. S.Sammito, I. Bockelmann, International Cardivascular Forum Journal 6, 18-22 (2016)

34. Susetyo, T. I. Oesman, S. T. Sudharman, Jurnal Teknologi (Institut Sains dan Teknologi AKPRIND Yogyakarta, 2012)

35. D.Wang, F. Dai, X. Ning, J. Constr. Eng. Manage. (2015)

36. L. A. Winanda, T. W. Adi, N. Anwar, Jurnal Ilmiah Teknik Sipil 21 (2), 99-107 (2017)

37. R. D. Wirahadikusumah, http://beta.lecture.ub.ac.id/files/2014/01/makalahreini-d-wirahadikusumah.pdf) (2016) (2014)

38. D. P. Wong, J. W. Chung, A. P. Chan, Appl. Ergon. 45, 1705-1711 (2014)

39. W. Yanfu, X. Min, 2012 International Symposium On Safety Science and Technology (pp. 131-138, 2012)

40. M. Zhang, E. H. Sparer, L. A. Murphy, J. T. Dennerlein, D. Fang, J. N. Katz, A. J. C. Martinez, Am. J. Ind. Med. 58 (2), 220-228 (2015)

41. D. G. Manolakis, V. K. Ingle, S. M. Kogon, Statistical and Adaptive Signal Processing: Spectral Estimation, Signal Modeling, Adaptive Filtering, and Array Processing, (McGraw-Hill, Boston, 2000)

42. S. V. Vasegi, Advanced digital Signal Processing and Noise Reduction Second Edition, (John Wiley \& Sons, London, 2000)

43. D. D Hastuti, Thesis, (Universitas Negeri Semarang, Semarang, 2015)

44. B. Bigband-Ritchie, F. Furbush, J. J. Woods, J. Appl. Physiol. 61, 421-429 (1986) 